Marijuana research gets backing at NIH

[WASHINGTON] A panel of scientists put together by the National Institutes of Health (NIH) said last week that the potential therapeutic uses of marijuana deserve further research and that - based on what they admitted to be scant data - the smoked drug is probably effective in some conditions.

"For at least some indications, there is a rationale for looking further into the therapeutic effects of marijuana," said William Beaver, a professor of pharmacology at Georgetown University in Washington DC, who chaired the panel.

The findings will add heat to the debate prompted by referendums last autumn in Arizona and California in which voters chose to legalize doctor-sanctioned smoking of marijuana for medicinal purposes. The US government has since threatened to prosecute doctors who prescribe the drug, a move that the New England Journal of Medicine has described as "misguided, heavy-handed and inhumane".

Separately, the Federation of American Scientists last month urged President Bill Clinton to instruct the NIH to carry out marijuana research, arguing that such research had been neglected "primarily for political reasons".

Last week's two-day workshop involved ten NIH institutes and divisions, and was led by the National Institute on Drug Abuse (NIDA). In a report to be completed next month, the eight-member panel that led the workshop will make formal recommendations on whether research on therapy using smoked marijuana should be pursued.

The panel's preliminary comments at a press conference at the end of the workshop were based on presentations by speakers who had reviewed hundreds of papers, mainly on the therapeutic value and clinical pharmacology of smoked marijuana and its major active ingredient, $\Delta$-9-tetrahydrocannabinol (THC). "It looks promising enough to recommend that there would be some new controlled studies," said Beaver. "Which ones? We're going to have to discuss this further."

At present the NIH - the only legal source of marijuana for experimental purposes does not fund any studies on the therapeutic use of marijuana, officials said. But last year NIDA, which supplies NIH institutes with the drug, provided marijuana for three studies on its metabolic impact and deleterious effects.

NIDA director Alan Leshner stressed NIH's "openness and willingness" to consider proposals from extramural investigators on marijuana as therapy. He said that the institutes had received just one such protocol in the past decade. It was rejected on scientific grounds. The study, proposed last year, would have examined marijuana's use in people with weight loss from AIDS.

\section{IMAGE \\ UNAVAILABLE FOR COPYRIGHT REASONS}

Herbal remedy? Cannabis indica in growth.

Beaver stressed that there is a lack of data on smoked marijuana as a therapeutic agent, although THC is a legally prescribed drug for nausea and vomiting in cancer chemotherapy and for appetite stimulation in wasting diseases including AIDS. Controlled studies of smoked marijuana face obstacles that include the difficulty of creating an effective placebo, the need to administer the drug at short intervals, and its long-term sideeffects.

MeredithWadman

\title{
High-level ethics committee 'needed to guide genetics policy'
}

[WASHINGTON] A panel of advisers to the US National Human Genome Research Institute (NHGRI) last week unanimously endorsed a proposal to create a high-profile committee to help guide administration policy on the ethical, legal and social implications of genome research.

The committee would operate in the office of the Secretary of Health and Human Services (HHS). It would take over at a higher level the role of the group that at present helps to shape policy on such issues within the institute, formerly the National Centre for Human Genome Research.

The reorganization was endorsed last Friday (21 February) by the National Advisory Council for Human Genome Research. It was proposed in a report prepared by an evaluation committee that was asked last spring by Francis Collins, director of the institute, to assess the role of the joint National Institutes of Health (NIH)/Department of Energy Working Group on the Ethical, Legal and Social Implications of Human Genome Research known as the ELSI working group.

The future of the working group had become controversial after Collins had said that budget cuts would mean that it would be able to meet only once a year (see Nature 381, 357;1996). Working- group members also complained that the genome research centre was muzzling it on controversial topics, and threatening its autonomy by establishing a separate Office of Policy Coordination.

The evaluation committee reported last December that the working group's mandate was "inadequately defined" and "much too broad" to be satisfied by a single body and that its low status as a subcommittee within the two departments "is not commensurate with the more global role of some important policy formulation".

The committee made three recommendations. First, the working group should be restructured as a 12-member ELSI Research Evaluation Committee, to advise Collins and his counterpart in the energy department on the quality, scope, direction and outcome of extramural ELSI research funded by NHGRI and the department.

Second, it urged that NIH director Harold Varmus should coordinate information and direction on ELSI projects within all $24 \mathrm{NIH}$ institutes, divisions and centres, to avoid duplication and to standardize research protocols.

Third, the committee proposed that responsibility for advising on and shaping government policy on ELSI issues should be shifted to a new independent Advisory Committee on Genetics and Public Policy in the office of the HHS secretary. This would provide a public forum for policy discussions on current and emerging ELSI issues, from health-insurance discrimination to the quality of genetic services.

Some members of the advisory council expressed concern about a proliferation of committees. Kurt Hirschhorn, a liaison member, said that he spoke for both the American Society of Human Genetics and the American College of Medical Genetics in stating "one real concern" about the proposed changes: the overlap of the HHSlevel Advisory Committee on Genetics and Public Policy with existing groups such as the National Bioethics Advisory Commission. He called instead for "a single bioethics national effort".

But at the end of last week's meeting the council endorsed the report's recommendations, on condition that NHGRI staff would work out details of the shape and function of the ELSI Research Evaluation Committee and present them to the council at its next meeting in May. 